\title{
Designing an Intuitionistic Fuzzy Network Data Envelopment Analysis Model for Efficiency Evaluation of Decision-Making Units with Two-Stage Structures
}

\author{
Nafiseh Javaherian $\mathbb{D}^{1},{ }^{1}$ Ali Hamzehee $\mathbb{D}^{1},{ }^{1}$ and Hossein Sayyadi Tooranloo ${ }^{2}$ \\ ${ }^{1}$ Department of Applied Mathematics, Kerman Branch, Islamic Azad University, Kerman, Iran \\ ${ }^{2}$ Department of Management, Meybod University, Meybod, Iran \\ Correspondence should be addressed to Ali Hamzehee; hamzehee_ali@yahoo.com
}

Received 26 June 2020; Revised 6 October 2020; Accepted 18 December 2020; Published 7 January 2021

Academic Editor: Jose A. Sanz

Copyright (c) 2021 Nafiseh Javaherian et al. This is an open access article distributed under the Creative Commons Attribution License, which permits unrestricted use, distribution, and reproduction in any medium, provided the original work is properly cited.

\begin{abstract}
Data envelopment analysis (DEA) is a powerful tool for evaluating the efficiency of decision-making units for ranking and comparison purposes and to differentiate efficient and inefficient units. Classic DEA models are ill-suited for the problems where decision-making units consist of multiple stages with intermediate products and those where inputs and outputs are imprecise or nondeterministic, which is not uncommon in the real world. This paper presents a new DEA model for evaluating the efficiency of decision-making units with two-stage structures and triangular intuitionistic fuzzy data. The paper first introduces two-stage DEA models, then explains how these models can be modified with intuitionistic fuzzy coefficients, and finally describes how arithmetic operators for intuitionistic fuzzy numbers can be used for a conversion into crisp two-stage structures. In the end, the proposed method is used to solve an illustrative numerical example.
\end{abstract}

\section{Introduction}

Data envelopment analysis is a standard quantitative tool with extensive use in efficiency evaluations and performance analysis [1]. DEA measures the relative efficiency of decision-making units (DMUs) with similar inputs and outputs in order to give an estimation of how efficient a unit is in comparison with other units [2-4].

However, most of the commonly used DEA models are criticized for treating units as black boxes and ignoring their internal processes, the efficiency of these processes, and their relationships $[5,6]$. This black box approach causes the analysis to miss a lot of valuable information about DMUs and limits its scope to the fundamental inputs and the ultimate outputs [7]. To address this issue, Färeet al. [8] introduced network data envelopment analysis (NDEA) and explained its importance for having a more accurate efficiency analysis of DMUs.

Unlike traditional DEA models, NDEA models have no fixed formulation and can be developed into different forms based on the type of process and network structure [9]. NDEA can very well illustrate the relationships and interdependencies between internal processes and accurately calculate the overall efficiency as well as the efficiency in each stage $[3,10]$. In addition, this method can be used for accurate tracking of the sources of inefficiency in inefficient units [11]. The two-stage network structure is one of the NDEA topologies that has been extensively studied by researchers [12-15].

Typically, data in DEA models are crisp and deterministic, but given the high frequency of uncertainties in real-world problems and impreciseness in real-world data, one simply cannot depend on classical mathematics to solve these problems. The solution to this issue is to use the gray dimension of classical logic, which is fuzzy logic, to improve the results of the models. The theory of fuzzy sets, which is an extended version of crisp sets, was first proposed by Zadeh [16], originally with the purpose of developing a more efficient model for use in natural language processing. 
Over the years, many researchers have used fuzzy logic in DEA models. For example, the works of Kao and Liu [17], Ramezanzadeh et al. [18], Saati et al. [19], Lertworasirikul et al. [20], Emrouznejad and Mustafa [21], Mirhedayatian et al. [22], Guo and Tanaka [23], Ghapanchi et al. [24], Sadeghi et al. [25], Rostamy-Malkhalifeh and Mollaeian [26], and Houshyar et al. [27] are some examples of studies conducted on DEA models with fuzzy data.

Following the development of fuzzy logic, Atanassov in 1986 [28] further expanded the theory of fuzzy sets to propose the intuitionistic fuzzy sets with three attributes: membership degree, nonmembership degree, and hesitation degree. Thus, intuitionistic fuzzy sets (IFSs) can efficiently model imperfect information, so to compare with fuzzy sets, IFSs are more effective in dealing with ambiguity and uncertainty [16, 29].

The use of fuzzy logic in other fields such as intuitionistic fuzzy DEA has been considered by researchers. For example, Arya and Yadav [30] developed intuitionistic fuzzy DEA models and their duals based on alpha-cuts and proposed an approach for finding intuitionistic fuzzy inputs and outputs in order to convert inefficient DMUs to efficient ones in an intuitionistic fuzzy environment. Puri and Yadav [31] presented an intuitionistic fuzzy DEA model where inputs and outputs are triangular intuitionistic fuzzy numbers. Otay et al. [32] used intuitionistic fuzzy sets to evaluate the efficiency of health and treatment centers in Istanbul, Turkey. Hajiagha et al. [33] presented weighted aggregation models for inputs and outputs with intuitionistic fuzzy data. Arya and Yadav [34] proposed a model called SBM, which is a nonradial DEA model for evaluating the efficiency of DMUs where inputs and outputs have intuitionistic fuzzy data. As the above review shows, despite many studies in this area, there has been no study on two-stage DEA in intuitionistic fuzzy environment. This study aims to expand the two-stage model of Chen et al. [35] for the intuitionistic fuzzy environment.

The two-stage model of Kao and Hwang [36] is an opposite model to consider the relationships between subprocesses and the overall process, and this model is certainly more logical than its precedents. However, this model cannot be extended to work under variable returns to scale (VRS) conditions, as it becomes nonlinear in these conditions. Chen et al. [35] modified this model into a similar model that is additive and can be used under both constant returns to scale (CRS) and VRS conditions. Also, unlike the model of Kao and Hwang [36] which cannot deal with intermediate variables with dual input/ output roles, the model of Chen et al. [35] can properly consider both roles of these variables. Therefore, this paper presents a new model, based on the two-stage model of Chen et al., for evaluating the efficiency of two-stage DMUs with intuitionistic fuzzy numbers under VRS conditions.

Therefore, the motivation of this present study is to develop two-stage DEA models in intuitionistic fuzzy environment with the variable returns to scale assumption based on the Chen et al. [35] model. Linearization of the nonlinear two-stage DEA models of intuitionistic fuzzy numbers that have computational complexity overcomes the limitations in using of these models in intuitionistic fuzzy environments. Another aim of this study is to linearize the proposed model with the expected value of intuitionistic fuzzy numbers.

\section{Preliminary}

2.1. Intuitionistic Fuzzy Set. Intuitionistic fuzzy set (IFS) is one of the generalizations from the fuzzy set theory [16]. Out of several higher-order fuzzy sets, IFS has been found to be more capable of dealing with vagueness. First introduced by Atanassov [37], IFS can be viewed as an alternative approach to conventional fuzzy set in dealing with cases with insufficient information. Fuzzy sets only consider the degree of acceptance, whereas IFS is characterized by both a membership function and a nonmembership function so that the sum of both values is less than one [28]. IFSs have been used across different fields of science, including the studies by Atanassov [28, 38-40], Szmidt and Kacprzyk [29], Buhaescu [41], Ban [42], Deschrijver and Kerre [43], and Stoyanova [44].

Definition 1 (see [28]). Assume $X$ is a reference set. In this case, set $\widetilde{A}^{I}$ which is a subset of $X$ is Atanassov's intuitionistic fuzzy set defined as follows:

$$
\tilde{A}^{I}=\left\{\prec x, \mu_{\sim_{A}^{I}}(x), v_{\widetilde{A}^{I}}(x) \succ, \forall x \in X\right\} .
$$

Such that, $\mu \sim I(x), v_{\sim_{I}}(x)$ are membership function and nonmembership function of $\widetilde{A}^{I}$, respectively, which are defined as $\mu \sim I(x): X \longrightarrow[0,1], v_{\sim I}(x): X \longrightarrow[0,1]$ and satisfy $0 \leq \mu_{\sim_{A}}(x)+v_{\sim_{A}}(x) \leq 1$. In addition, for each $x \in X$, intuitionistic index or the hesitancy degree of $x, \pi_{x}$ is defined as $\pi_{x}=1-\mu_{A}^{I}(x)-v_{A}(x)$.

\subsubsection{Intuitionistic Fuzzy Number}

Definition 2 (see [32]). Let $\widetilde{A}^{I}$ be an IFS in $X$; then, $\widetilde{A}^{I}$ is said to be an intuitionistic fuzzy number (IFN), if

(1) it is normal, i.e., $\forall x_{\circ} \in X: \mu_{A}\left(x_{\circ}\right)=1, v_{\widetilde{A}^{I}}\left(x_{\mathrm{o}}\right)=0$

(2) $\mu_{A} I(x)$ is convex, i.e., $\quad \mu_{A}^{I}\left(\lambda x_{1}+(1-\lambda) x_{2}\right) \geq$ $\min \left\{\mu_{A}\left(x_{1}\right), \mu_{A} I\left(x_{2}\right)\right\}, \forall x_{1}, x_{2} \in X, \lambda \in[0,1]$

(3) $v_{\sim_{A}^{I}}(x)$ is concave, i.e., $v_{\sim_{A}}\left(\lambda x_{1}+(1-\lambda) x_{2}\right) \leq$ $\max \left\{v_{\widetilde{A}^{I}}\left(x_{1}\right), v_{\widetilde{A}^{I}}\left(x_{2}\right)\right\}, \forall x_{1}, x_{2} \in X, \lambda \in[0,1]$

Definition 3. An IFN can be named $\prec x, \mu_{A} I(x), v_{\sim_{A}}(x) \succ=\left(a^{1}, a^{2}, a^{3}, a^{4} ; b^{1}, b^{2}, b^{3}, b^{4}\right)$, such that degrees of membership $\mu_{A}^{A}(x)$ and nonmembership $v_{\sim_{I} I}(x)$ are as follows:

$$
\begin{aligned}
& \mu_{\sim_{A}^{I}}(x)= \begin{cases}f_{A}(x), & a^{1} \leq x<a^{2}, \\
1, & a^{2} \leq x \leq a^{3}, \\
g_{A}(x), & a^{3}<x \leq a^{4}, \\
0, & \text { otherwise, }\end{cases} \\
& v_{\widetilde{A}^{I}}(x)= \begin{cases}h_{A}(x), & b^{1} \leq x<b^{2}, \\
0, & b^{2} \leq x \leq b^{3}, \\
k_{A}(x), & b^{3} \leq x<b^{4}, \\
1, & \text { otherwise, }\end{cases}
\end{aligned}
$$


where $f_{A}, k_{A}$ are monotonically increasing functions and $g_{A}, h_{A}$ are monotonically decreasing functions.

2.1.2. Triangular Intuitionistic Fuzzy Number. Triangular intuitionistic fuzzy number (TIFN) is a useful tool in expressing ill-known quantities [45].

Definition 4 (see [13]). An IFN is said to be a TIFN if its membership function $\left(\mu_{\sim}(x)\right)$ and its nonmembership function $\left(v_{\sim_{A}^{I}}(x)\right)$ are as follows:

$$
\begin{aligned}
& \mu_{A} I(x)= \begin{cases}\frac{x-a^{1}}{a^{2}-a^{1}}, & a^{1}<x \leq a^{2}, \\
1, & x=a^{2}, \\
\frac{x-a^{3}}{a^{2}-a^{3}}, & a^{2} \leq x<a^{3}, \\
0, & \text { otherwise, }\end{cases} \\
& v_{\sim^{I}}(x)= \begin{cases}\frac{x-a^{2}}{a^{1^{\prime}}-a^{2}}, & a^{1^{\prime}} \leq x \leq a^{2}, \\
0, & x=a^{2}, \\
\frac{x-a^{2}}{a^{3^{\prime}}-a^{3}}, & a^{2} \leq x \leq a^{3^{\prime}}, \\
1, & \text { otherwise, }\end{cases}
\end{aligned}
$$

where $a^{1^{\prime}} \leq a^{1} \leq a^{2} \leq a^{3} \leq a^{3^{\prime}}$.

This TIFN is shown in the form of $\tilde{A}^{I}=$ $\left(a^{1}, a^{2}, a^{3} ; a^{1^{\prime}}, a^{2}, a^{3^{\prime}}\right)$.

2.1.3. Arithmetic Operations on Intuitionistic Fuzzy Numbers. Let $\quad \widetilde{A}^{I}=\left(a^{1}, a^{2}, a^{3} ; a^{1^{1}}, a^{2}, a^{3^{1}}\right)$ and $\widetilde{B}^{I}=$ $\left(b^{1}, b^{2}, b^{3} ; b^{1^{\prime}}, b^{2}, b^{3^{\prime}}\right)$ be TIFNs, then the following relationships hold:

(i) The addition of $\widetilde{A}^{I}$ and $\widetilde{B}^{I}$ is $\widetilde{A}^{I} \oplus \widetilde{B}^{I}=\left(a^{1}+b^{1}, a^{2}+b^{2}, a^{3}+b^{3} ; a^{1^{\prime}}+b^{1^{\prime}}, a^{2}+b^{2}, a^{3^{\prime}}+b^{3^{\prime}}\right)$,

(ii) The product of $\widetilde{A}^{I}$ and $\widetilde{B}^{I}$ is $\widetilde{A}^{I} \otimes \widetilde{B}^{I} \approx\left(a^{1} b^{1}, a^{2} b^{2}, a^{3} b^{3} ; a^{1^{\prime}} b^{1^{\prime}}, a^{2} b^{2}, a^{3^{\prime}} b^{3^{\prime}}\right)$,

(iii) For every $k \in R ; k \widetilde{A}^{I}= \begin{cases}\left(k a^{1}, k a^{2}, k a^{3} ; k a^{1^{\prime}}, k a^{2}, k a^{3^{\prime}}\right), & \text { if } k>0, \\ \left(k a^{3}, k a^{2}, k a^{1} ; k a^{3^{\prime}}, k a^{2}, k a^{1^{\prime}}\right), & \text { if } k<0 .\end{cases}$

2.1.4. Expected Values of Intuitionistic Fuzzy Numbers and Their Characteristics
Definition 5 (see [46]). Let $\widetilde{A}^{I}=\left(a^{1}, a^{2}, a^{3}, a^{4} ; b^{1}, b^{2}, b^{3}, b^{4}\right)$ be an IFN, then the expected interval is defined, as follows: 


$$
E I\left(\widetilde{A}^{I}\right)=\left[E_{L}\left(\tilde{A}^{I}\right), E_{R}\left(\tilde{A}^{I}\right)\right]
$$

where $E_{L}\left(\tilde{A}^{I}\right)=\frac{b^{1}+a^{2}}{2}+\frac{1}{2} \int_{b^{1}}^{b^{2}} h_{A}(x)-\frac{1}{2} \int_{a^{1}}^{a^{2}} f_{A}(x) \mathrm{d} x$,

$$
\text { and } E_{R}\left(\tilde{A}^{I}\right)=\frac{a^{3}+b^{4}}{2}+\frac{1}{2} \int_{a^{3}}^{a^{4}} g_{A}(x)-\frac{1}{2} \int_{b^{3}}^{b^{4}} k_{A}(x) \mathrm{d} x \text {. }
$$

Accordingly, the expected value based on this IFN is defined as

$$
E V\left(\widetilde{A}^{I}\right)=\frac{\left(E_{L}\left(\tilde{A}^{I}\right)+E_{R}\left(\tilde{A}^{I}\right)\right)}{2} .
$$

Proposition 1. If $\tilde{A}^{I}=\left(a^{1}, a^{2}, a^{3} ; a^{1^{\prime}}, a^{2}, a^{3^{\prime}}\right)$ be a TIFN, then

$$
\begin{aligned}
& \text { (i) } E I\left(\widetilde{A}^{I}\right)=\left[\frac{a^{1^{\prime}}+2 a^{2}+a^{1}}{4}, \frac{a^{3}+2 a^{2}+a^{3^{\prime}}}{4}\right] \text {, } \\
& \text { (ii) } E V\left(\widetilde{A}^{I}\right)=\frac{a^{1^{\prime}}+a^{1}+4 a^{2}+a^{3}+a^{3^{\prime}}}{8} .
\end{aligned}
$$

Proof. According to Definitions 3-5,

$$
E_{L}\left(\widetilde{A}^{I}\right)=\frac{a^{1^{\prime}}+a^{2}}{2}+\frac{1}{2} \int_{a^{1^{1}}}^{a^{2}} \frac{x-a^{2}}{a^{1^{\prime}}-a^{2}}-\frac{1}{2} \int_{a^{1}}^{a^{2}} \frac{x-a^{1}}{a^{2}-a^{1}} \mathrm{~d} x .
$$

Firstly,

$$
\begin{aligned}
\int_{a^{1^{1}}}^{a^{2}} \frac{x-a^{2}}{a^{1^{\prime}}-a^{2}} \mathrm{~d} x & =\frac{1}{a^{1^{\prime}}-a^{2}}\left(\frac{x^{2}}{2}-a^{2} x\right)=\frac{1}{a^{1^{\prime}}-a^{2}}\left(\frac{\left(a^{2}\right)^{2}}{2}-\left(a^{2}\right)^{2}-\frac{\left(a^{1^{\prime}}\right)^{2}}{2}+a^{1^{\prime}} a^{2}\right)=\frac{1}{a^{1^{\prime}}-a^{2}}\left(-\frac{\left(a^{2}\right)^{2}}{2}-\frac{\left(a^{1^{\prime}}\right)^{2}}{2}+a^{1^{\prime}} a^{2}\right) \\
& =\frac{1}{a^{1^{\prime}}-a^{2}}\left(-\frac{1}{2}\left(a^{1^{\prime}}-a^{2}\right)^{2}\right)=-\frac{1}{2}\left(a^{1^{\prime}}-a^{2}\right) .
\end{aligned}
$$

Secondly,

$$
\int_{a^{1}}^{a^{2}} \frac{x-a^{1}}{a^{2}-a^{1}} \mathrm{~d} x=\frac{1}{a^{2}-a^{1}}\left(\frac{x^{2}}{2}-a^{1} x\right)=\frac{1}{a^{2}-a^{1}}\left(\frac{\left(a^{2}\right)^{2}}{2}-a^{1} a^{2}-\frac{\left(a^{1}\right)^{2}}{2}+\left(a^{1}\right)^{2}\right)=\frac{1}{a^{2}-a^{1}}\left(\frac{1}{2}\left(a^{2}-a^{1}\right)^{2}\right)=\frac{1}{2}\left(a^{2}-a^{1}\right) .
$$

So,

$$
E_{L}\left(\widetilde{A}^{I}\right)=\frac{a^{1^{\prime}}+a^{2}}{2}-\frac{1}{4}\left(a^{1^{\prime}}-a^{2}\right)-\frac{1}{4}\left(a^{2}-a^{1}\right)=\frac{a^{1^{\prime}}+2 a^{2}+a^{1}}{4} .
$$

With this process, $E_{R}\left(\widetilde{A}^{I}\right)=\left(\left(a^{3}+2 a^{2}+a^{3^{\prime}}\right) / 4\right)$ and $E V\left(\widetilde{A}^{I}\right)$ are proved too.

Remark 1. The expected value is a linear operator, meaning that for any two IFNs such as $\widetilde{A}^{I}$ and $\widetilde{B}^{I}$, the following relationship holds:

$$
E V\left(\widetilde{A}^{I}+\widetilde{B}^{I}\right)=E V\left(\widetilde{A}^{I}\right)+E V\left(\widetilde{B}^{I}\right) .
$$

\subsubsection{Accuracy Function of TIFNs}

Definition 6. (see [47]). Let $\widetilde{A}^{I}=\left(a^{1}, a^{2}, a^{3} ; a^{1^{\prime}}, a^{2}, a^{3^{\prime}}\right)$ be a TIFN. The score function for the membership function $\mu \sim I(x)$ is denoted by $S(\mu \sim I)$ and is defined by $S\left(\underset{A}{A} \sim_{I}\right)=\left(\left(a^{1}+2 a^{2}+a^{3}\right) / 4\right)$. The score function for the nonmembership function $v_{\sim_{I}}(x)$ is denoted by $S\left(v_{\sim_{A}}\right)$ and is defined by $S(v \sim I)=\left(a^{1^{\prime}}+\stackrel{A}{a} a^{2}+a^{3^{\prime}} / 4\right)$. The accuracy function of $\widetilde{A}^{I}$ is denoted by $f\left(\widetilde{A}^{I}\right)$ and defined by

$$
\begin{aligned}
f\left(\tilde{A}^{I}\right) & =\frac{S\left(\mu_{\sim^{I}}\right)+S\left(\nu_{\widetilde{A}^{I}}\right)}{2} \\
& =\frac{\left(a^{1}+2 a^{2}+a^{3}\right)+\left(a^{1^{\prime}}+2 a^{2}+a^{3^{\prime}}\right)}{8} .
\end{aligned}
$$

2.2. Network Data Envelopment Analysis and Two-Stage Structures. DEA is one of the most effective methods for efficiency evaluation and comparison of decision-making units. The development of the DEA literature has led to the 
emergence of several different models for efficiency assessment, and one of which is the DEA with a network structure. In these models, the production process consists of multiple stages, which are linked together and to their inputs, intermediate products, and outputs via a set of processes. The two-stage structure was first introduced by Färe [9] and later expanded in other works [8, 48, 49]. Färe and Grosskopf also [49] formulated the relationships between various production processes in the framework of NDEA, where the hierarchical structure of activities is replaced with a network structure [11]. At first, these structures were designed so that the outputs of the first stage were the only inputs of the second stage. However, later, the two-stage network structures were expanded to allow the second stage to take other inputs on top of the outputs of the first stage. Efficiency evaluation of two-stage systems is one of the interesting topics in the field of DEA.

\section{Materials and Methods}

3.1. Development of the Two-Stage DEA Model in the Intuitionistic Fuzzy Environment. As mentioned, the present study aims to expand the model of Chen et al. [35] for an intuitionistic fuzzy environment. Therefore, first, the model of Chen et al. should be briefly introduced. Suppose there are $n$ numbers of two-stage decision-making units of the form $\mathrm{DMU}_{j},(j=1, \ldots, n)$ that need to be evaluated. In the first stage, each $\mathrm{DMU}_{j}$ takes $m$ inputs of the form $X_{i j},(i=1, \ldots, m)$ to produce $D$ outputs of the form $Z_{d j},(d=1, \ldots, D)$, which are considered intermediate products and will be the inputs of the second stage. The second stage produces $s$ outputs in the form of $Y_{r j},(r=1, \ldots, s)$. According to the model of Chen et al. [35], the overall efficiency of this unit under the VRS assumption is as follows:

$$
\begin{aligned}
E_{\circ}= & \max \sum_{r=1}^{s} \mu_{r} y_{r j o}+u^{1}+\sum_{d=1}^{D} \pi_{d} z_{d j \circ}+u^{2} \\
\text { s.t. } & \sum_{d=1}^{D} \pi_{d} z_{d j}-\sum_{i=1}^{m} \omega_{i} x_{i j}+u^{1} \leq 0, \quad j=1,2, \ldots, n, \\
& \sum_{r=1}^{s} \mu_{r} y_{r j}-\sum_{d=1}^{D} \pi_{d} z_{d j}+u^{2} \leq 0, \quad j=1,2, \ldots, n, \\
& \sum_{i=1}^{m} \omega_{i} x_{i j \circ}+\sum_{d=1}^{D} \pi_{d} z_{d j \circ}=1, \quad j=1,2, . ., n, \\
& \pi_{d}, \mu_{r}, \omega_{i} \geq 0, \quad d=1, \ldots, D ; r=1, \ldots, s ; i=1, \ldots, m, \\
& u^{1}, u^{2} \text { free in sign, }
\end{aligned}
$$

where $u^{1}, u^{2}$ are unrestricted in sign, and their sign defines the returns to scale, as follows:

(A) If $u^{1}, u^{2}<0$, then returns to scale is increasing

(B) If $u^{1}, u^{2}>0$, then returns to scale is decreasing
(C) If $u^{1}, u^{2}=0$, then returns to scale is constant

If the variables are represented by IFNs, then model (15) can be rewritten, as follows:

$$
\begin{aligned}
& \widetilde{E}_{\circ}^{I}=\max \sum_{r=1}^{s} \tilde{\mu}_{r}^{I} \otimes \tilde{y}_{r j \circ}^{I}+u^{1} \oplus \sum_{d=1}^{D} \tilde{\pi}_{d}^{I} \otimes \widetilde{z}_{d j \circ}^{I}+u^{2} \\
& \text { s.t. } \sum_{d=1}^{D} \tilde{\pi}_{d}^{I} \otimes \widetilde{z}_{d j}^{I} \Theta \sum_{i=1}^{m} \widetilde{\omega}_{i}^{I} \otimes \widetilde{x}_{i j}^{I}+u^{1} \leq \widetilde{0}^{I}, \quad j=1,2, \ldots, n \text {, } \\
& \sum_{r=1}^{s} \tilde{\mu}_{r}^{I} \otimes \tilde{y}_{r j}^{I} \Theta \sum_{d=1}^{D} \tilde{\pi}_{d}^{I} \otimes \widetilde{z}_{d j}^{I}+u^{2} \leq \widetilde{0}^{I}, \quad j=1,2, \ldots, n, \\
& \sum_{i=1}^{m} \widetilde{\omega}_{i}^{I} \otimes \widetilde{x}_{i j \circ}^{I} \oplus \sum_{d=1}^{D} \tilde{\pi}_{d}^{I} \otimes \widetilde{z}_{d j \circ}^{I}=\tilde{1}^{I}, \quad j=1,2, \ldots, n, \\
& \tilde{\pi}_{d}^{I}, \tilde{\mu}_{r}^{I}, \widetilde{\omega}_{i}^{I} \geq \varepsilon>0, \quad d=1, \ldots, D ; r=1, \ldots, s ; i=1, \ldots, m, \\
& u^{1}, u^{2} \text { free in sign. }
\end{aligned}
$$


Here, $\varepsilon$ is a small number that is used to prevent giving a weight of zero to the undesirable factors of the DMU under evaluation.
The aim of this study is to expand the above two-stage DEA model with TIFNs. Therefore, model (16) is rewritten based on TIFNs and the method of Puri and Yadav [31] to obtain

$$
\begin{aligned}
& \widetilde{E}_{\circ}^{I}=\max \sum_{r=1}^{s}\left(\mu_{r}^{1}, \mu_{r}^{2}, \mu_{r}^{3} ; \mu_{r}^{1^{\prime}}, \mu_{r}^{2}, \mu_{r}^{3^{\prime}}\right) \otimes\left(y_{r j \circ}^{1}, y_{r j \circ}^{2}, y_{r j \circ}^{3} ; y_{r j \circ}^{1^{\prime}}, y_{r j \circ}^{2}, y_{r j \circ}^{3^{\prime}}\right)+u^{1} \oplus \sum_{d=1}^{D}\left(\pi_{d}^{1}, \pi_{d}^{2}, \pi_{d}^{3} ; \pi_{d}^{1^{\prime}}, \pi_{d}^{2}, \pi_{d}^{3^{\prime}}\right) \\
& \otimes\left(z_{d j \circ}^{1}, z_{d j \circ}^{2}, z_{d j \circ}^{3} ; z_{d j \circ}^{1^{\prime}}, z_{d j \circ}^{2}, z_{d j \circ}^{3^{\prime}}\right)+u^{2} \\
& \text { s.t. } \quad \sum_{d=1}^{D}\left(\pi_{d}^{1}, \pi_{d}^{2}, \pi_{d}^{3} ; \pi_{d}^{1^{\prime}}, \pi_{d}^{2}, \pi_{d}^{3^{\prime}}\right) \otimes\left(z_{d j}^{1}, z_{d j}^{2}, z_{d j}^{3} ; z_{d j}^{1^{\prime}}, z_{d j}^{2}, z_{d j}^{3^{\prime}}\right) \Theta \sum_{i=1}^{m}\left(\omega_{i}^{1}, \omega_{i}^{2}, \omega_{i}^{3} ; \omega_{i}^{1^{\prime}}, \omega_{i}^{2}, \omega_{i}^{3^{\prime}}\right) \\
& \otimes\left(x_{i j}^{1}, x_{i j}^{2}, x_{i j}^{3} ; x_{i j}^{1^{\prime}}, x_{i j}^{2}, x_{i j}^{3^{\prime}}\right)+u^{1} \leq(0,0,0 ; 0,0,0), \quad j=1,2, \ldots, n, \\
& \sum_{r=1}^{s}\left(\mu_{r}^{1}, \mu_{r}^{2}, \mu_{r}^{3} ; \mu_{r}^{1^{\prime}}, \mu_{r}^{2}, \mu_{r}^{3^{\prime}}\right) \otimes\left(y_{r j}^{1}, y_{r j}^{2}, y_{r j}^{3} ; y_{r j}^{1^{\prime}}, y_{r j}^{2}, y_{r j}^{3^{\prime}}\right) \Theta \sum_{d=1}^{D}\left(\pi_{d}^{1}, \pi_{d}^{2}, \pi_{d}^{3} ; \pi_{d}^{1^{\prime}}, \pi_{d}^{2}, \pi_{d}^{3^{\prime}}\right) \\
& \otimes\left(z_{d j}^{1}, z_{d j}^{2}, z_{d j}^{3} ; z_{d j}^{1^{\prime}}, z_{d j}^{2}, z_{d j}^{3^{\prime}}\right)+u^{2} \leq(0,0,0 ; 0,0,0), \quad j=1,2, \ldots, n, \\
& \sum_{d=1}^{D}\left(\pi_{d}^{1}, \pi_{d}^{2}, \pi_{d}^{3} ; \pi_{d}^{1^{\prime}}, \pi_{d}^{2}, \pi_{d}^{3^{\prime}}\right) \otimes\left(z_{d j}^{1}, z_{d j}^{2}, z_{d j}^{3} ; z_{d j}^{1^{\prime}}, z_{d j}^{2}, z_{d j}^{3^{\prime}}\right)+u^{2} \leq(0,0,0 ; 0,0,0), \quad j=1,2, \ldots, n, \\
& \sum_{d=1}^{D}\left(\pi_{d}^{1}, \pi_{d}^{2}, \pi_{d}^{3} ; \pi_{d}^{1^{\prime}}, \pi_{d}^{2}, \pi_{d}^{3^{\prime}}\right) \otimes\left(z_{d j}^{1}, z_{d j}^{2}, z_{d j}^{3} ; z_{d j}^{1^{\prime}}, z_{d j}^{2}, z_{d j}^{3^{\prime}}\right)+u^{2} \leq(0,0,0 ; 0,0,0), \quad j=1,2, \ldots, n, \\
& \sum_{d=1}^{D}\left(\pi_{d}^{1}, \pi_{d}^{2}, \pi_{d}^{3} ; \pi_{d}^{1^{\prime}}, \pi_{d}^{2}, \pi_{d}^{3^{\prime}}\right) \otimes\left(z_{d j}^{1}, z_{d j}^{2}, z_{d j}^{3} ; z_{d j}^{1^{\prime}}, z_{d j}^{2}, z_{d j}^{3^{\prime}}\right)+u^{2} \leq(0,0,0 ; 0,0,0), \quad j=1,2, \ldots, n \\
& \sum_{d=1}^{D}\left(\pi_{d}^{1}, \pi_{d}^{2}, \pi_{d}^{3} ; \pi_{d}^{1^{\prime}}, \pi_{d}^{2}, \pi_{d}^{3^{\prime}}\right) \otimes\left(z_{d j \circ}^{1}, z_{d j \circ}^{2}, z_{d j \circ}^{3} ; z_{d j \circ}^{1^{\prime}}, z_{d j \circ}^{2}, z_{d j \circ}^{3^{\prime}}\right)=(1,1,1 ; 1,1,1), \quad j=1,2, \ldots, n, \\
& \left(\pi_{d}^{1}, \pi_{d}^{2}, \pi_{d}^{3} ; \pi_{d}^{1^{\prime}}, \pi_{d}^{2}, \pi_{d}^{3^{\prime}}\right) \geq \varepsilon>0, \quad d=1, \ldots, D, \\
& \left(\mu_{r}^{1}, \mu_{r}^{2}, \mu_{r}^{3} ; \mu_{r}^{1^{\prime}}, \mu_{r}^{2}, \mu_{r}^{3^{\prime}}\right) \geq \varepsilon>0, \quad r=1, \ldots, s, \\
& \left(\omega_{i}^{1}, \omega_{i}^{2}, \omega_{i}^{3} ; \omega_{i}^{1^{\prime}}, \omega_{i}^{2}, \omega_{i}^{3^{\prime}}\right) \geq \varepsilon>0, \quad i=1, \ldots, m, \\
& u^{1}, u^{2} \text { free in sign. }
\end{aligned}
$$


Using the arithmetic operations described in relations (4) for TIFNs, model (17) is rewritten into the following form:

$$
\begin{aligned}
& \widetilde{E}_{\circ}^{I}=\max \left(\sum_{r=1}^{s} \mu_{r}^{1} y_{r j \circ}^{1}+\sum_{d=1}^{D} \pi_{d}^{1} z_{d j \circ}^{1}, \sum_{r=1}^{s} \mu_{r}^{2} y_{r j \circ}^{2}+\sum_{d=1}^{D} \pi_{d}^{2} z_{d j \circ}^{2}, \sum_{r=1}^{s} \mu_{r}^{3} y_{r j \circ}^{3}+\sum_{d=1}^{D} \pi_{d}^{3} z_{d j \circ}^{3} ; \sum_{r=1}^{s} \mu_{r}^{1^{\prime}} y_{r j \circ}^{1^{\prime}}+\sum_{d=1}^{D} \pi_{d}^{1^{\prime}} z_{d j \circ}^{1^{\prime}}, \sum_{r=1}^{s} \mu_{r}^{2} y_{r j \circ}^{2}\right. \\
& \left.+\sum_{d=1}^{D} \pi_{d}^{2} z_{d j \circ}^{2}, \sum_{r=1}^{s} \mu_{r}^{3^{\prime}} y_{r j \circ}^{3^{\prime}}+\sum_{d=1}^{D} \pi_{d}^{3^{\prime}} z_{d j \circ}^{3^{\prime}}\right)+u^{1}+u^{2} \\
& \text { s.t. } \quad\left(\sum_{d=1}^{D} \pi_{d}^{1} z_{d j}^{1}-\sum_{i=1}^{m} \omega_{i}^{3} x_{i j}^{3}, \sum_{d=1}^{D} \pi_{d}^{2} z_{d j}^{2}-\sum_{i=1}^{m} \omega_{i}^{2} x_{i j}^{2}, \sum_{d=1}^{D} \pi_{d}^{3} z_{d j}^{3}-\sum_{i=1}^{m} \omega_{i}^{1} x_{i j}^{1} ; \sum_{d=1}^{D} \pi_{d}^{1^{\prime}} z_{d j}^{1^{\prime}}-\sum_{i=1}^{m} \omega_{i}^{3^{\prime}} x_{i j}^{3^{\prime}}, \sum_{d=1}^{D} \pi_{d}^{2} z_{d j}^{2}\right. \\
& \left.-\sum_{i=1}^{m} \omega_{i}^{2} x_{i j}^{2}, \sum_{d=1}^{D} \pi_{d}^{3^{\prime}} z_{d j}^{3^{\prime}}-\sum_{i=1}^{m} \omega_{i}^{1^{\prime}} x_{i j}^{1^{\prime}}\right)+u^{1} \leq(0,0,0 ; 0,0,0), \quad j=1,2, \ldots, n, \\
& \left(\sum_{r=1}^{s} \mu_{r}^{1} y_{r j}^{1}-\sum_{d=1}^{D} \pi_{d}^{1} z_{d j}^{1}, \sum_{r=1}^{s} \mu_{r}^{2} y_{r j}^{2}-\sum_{d=1}^{D} \pi_{d}^{2} z_{d j}^{2}, \sum_{r=1}^{s} \mu_{r}^{3} y_{r j}^{3}-\sum_{d=1}^{D} \pi_{d}^{3} z_{d j}^{3} ; \sum_{r=1}^{s} \mu_{r}^{1^{\prime}} y_{r j}^{1^{\prime}}-\sum_{d=1}^{D} \pi_{d}^{1^{\prime}} z_{d j}^{1^{\prime}}, \sum_{r=1}^{s} \mu_{r}^{2} y_{r j}^{2}\right. \\
& \left.-\sum_{d=1}^{D} \pi_{d}^{2} z_{d j}^{2}, \sum_{r=1}^{s} \mu_{r}^{3^{\prime}} y_{r j}^{3^{\prime}}-\sum_{d=1}^{D} \pi_{d}^{3^{\prime}} z_{d j}^{3^{\prime}}\right)+u^{2} \leq(0,0,0 ; 0,0,0), \quad j=1,2, \ldots, n \\
& \left(\sum_{i=1}^{m} \omega_{i}^{1} x_{i j \circ}^{1}+\sum_{d=1}^{D} \pi_{d}^{1} z_{d j \circ}^{1}, \sum_{i=1}^{m} \omega_{i}^{2} x_{i j \circ}^{2}+\sum_{d=1}^{D} \pi_{d}^{2} z_{d j \circ}^{2}, \sum_{i=1}^{m} \omega_{i}^{3} x_{i j \circ}^{3}+\sum_{d=1}^{D} \pi_{d}^{3} z_{d j \circ}^{3} ; \sum_{i=1}^{m} \omega_{i}^{1^{\prime}} x_{i j \circ}^{1^{\prime}}+\sum_{d=1}^{D} \pi_{d}^{1^{\prime}} z_{d j \circ}^{1^{\prime}}, \sum_{i=1}^{m} \omega_{i}^{2} x_{i j \circ}^{2}\right. \\
& \left.+\sum_{d=1}^{D} \pi_{d}^{2} z_{d j \circ}^{2}, \sum_{i=1}^{m} \omega_{i}^{3^{\prime}} x_{i j \circ}^{3^{\prime}}+\sum_{d=1}^{D} \pi_{d}^{3^{\prime}} z_{d j \circ}^{3^{\prime}}\right)=(1,1,1 ; 1,1,1), \quad j=1,2, \ldots, n \\
& \left(\pi_{d}^{1}, \pi_{d}^{2}, \pi_{d}^{3} ; \pi_{d}^{1^{\prime}}, \pi_{d}^{2}, \pi_{d}^{3^{\prime}}\right) \geq \varepsilon>0, \quad d=1, \ldots, D, \\
& \left(\mu_{r}^{1}, \mu_{r}^{2}, \mu_{r}^{3} ; \mu_{r}^{1^{\prime}}, \mu_{r}^{2}, \mu_{r}^{3^{\prime}}\right) \geq \varepsilon>0, \quad r=1, \ldots, s, \\
& \left(\omega_{i}^{1}, \omega_{i}^{2}, \omega_{i}^{3} ; \omega_{i}^{1^{\prime}}, \omega_{i}^{2}, \omega_{i}^{3^{\prime}}\right) \geq \varepsilon>0, \quad i=1, \ldots, m, \\
& u^{1}, u^{2} \text { free in sign. }
\end{aligned}
$$

As the coefficients of model (18) show, this model is represented by IFNs with 6 components. Therefore, this model is converted to a linear crisp (nonfuzzy) model based on the expected value of IFNs. After determining the expected value based on the objective function and the constraints of model (18), model (19) will be obtained, as follows: 


$$
\begin{aligned}
& E V\left(\widetilde{E}_{\circ}^{I}\right)=\max E V\left(\left(\sum_{r=1}^{s} \mu_{r}^{1} y_{r j \circ}^{1}+\sum_{d=1}^{D} \pi_{d}^{1} z_{d j \circ}^{1}, \sum_{r=1}^{s} \mu_{r}^{2} y_{r j \circ}^{2}+\sum_{d=1}^{D} \pi_{d}^{2} z_{d j \circ}^{2}, \sum_{r=1}^{s} \mu_{r}^{3} y_{r j \circ}^{3}+\sum_{d=1}^{D} \pi_{d}^{3} z_{d j \circ}^{3} ; \sum_{r=1}^{s} \mu_{r}^{1^{\prime}} y_{r j \circ}^{1^{\prime}}\right.\right. \\
& \left.\left.+\sum_{d=1}^{D} \pi_{d}^{1^{\prime}} z_{d j \circ}^{1^{\prime}}, \sum_{r=1}^{s} \mu_{r}^{2} y_{r j \circ}^{2}+\sum_{d=1}^{D} \pi_{d}^{2} z_{d j \circ}^{2}, \sum_{r=1}^{s} \mu_{r}^{3^{\prime}} y_{r j \circ}^{3^{\prime}}+\sum_{d=1}^{D} \pi_{d}^{3^{\prime}} z_{d j \circ}^{3^{\prime}}\right)+u^{1}+u^{2}\right), \\
& \text { s.t. } \quad E V\left(\left(\sum_{d=1}^{D} \pi_{d}^{1} z_{d j}^{1}-\sum_{i=1}^{m} \omega_{i}^{3} x_{i j}^{3}, \sum_{d=1}^{D} \pi_{d}^{2} z_{d j}^{2}-\sum_{i=1}^{m} \omega_{i}^{2} x_{i j}^{2}, \sum_{d=1}^{D} \pi_{d}^{3} z_{d j}^{3}-\sum_{i=1}^{m} \omega_{i}^{1} x_{i j}^{1} ; \sum_{d=1}^{D} \pi_{d}^{1^{\prime}} z_{d j}^{1^{\prime}}-\sum_{i=1}^{m} \omega_{i}^{3^{\prime}} x_{i j}^{3^{\prime}}, \sum_{d=1}^{D} \pi_{d}^{2} z_{d j}^{2}\right.\right. \\
& \left.\left.-\sum_{i=1}^{m} \omega_{i}^{2} x_{i j}^{2}, \sum_{d=1}^{D} \pi_{d}^{3^{\prime}} z_{d j}^{3^{\prime}}-\sum_{i=1}^{m} \omega_{i}^{1^{\prime}} x_{i j}^{1^{\prime}}\right)+u^{1}\right) \leq E V((0,0,0 ; 0,0,0)), \quad j=1,2, \ldots, n, \\
& E V\left(\left(\sum_{r=1}^{s} \mu_{r}^{1} y_{r j}^{1}-\sum_{d=1}^{D} \pi_{d}^{1} z_{d j}^{1}, \sum_{r=1}^{s} \mu_{r}^{2} y_{r j}^{2}-\sum_{d=1}^{D} \pi_{d}^{2} z_{d j}^{2}, \sum_{r=1}^{s} \mu_{r}^{3} y_{r j}^{3}-\sum_{d=1}^{D} \pi_{d}^{3} z_{d j}^{3} ; \sum_{r=1}^{s} \mu_{r}^{1^{\prime}} y_{r j}^{1^{\prime}}-\sum_{d=1}^{D} \pi_{d}^{1^{\prime}} z_{d j}^{1^{\prime}}, \sum_{r=1}^{s} \mu_{r}^{2} y_{r j}^{2}\right.\right. \\
& \left.\left.-\sum_{d=1}^{D} \pi_{d}^{2} z_{d j}^{2}, \sum_{r=1}^{s} \mu_{r}^{3^{\prime}} y_{r j}^{3^{\prime}}-\sum_{d=1}^{D} \pi_{d}^{3^{\prime}} z_{d j}^{3^{\prime}}\right)+u^{2}\right) \leq E V((0,0,0 ; 0,0,0)), \quad j=1,2, \ldots, n, \\
& E V\left(\left(\sum_{i=1}^{m} \omega_{i}^{1} x_{i j \circ}^{1}+\sum_{d=1}^{D} \pi_{d}^{1} z_{d j \circ}^{1}, \sum_{i=1}^{m} \omega_{i}^{2} x_{i j \circ}^{2}+\sum_{d=1}^{D} \pi_{d}^{2} z_{d j \circ}^{2}, \sum_{i=1}^{m} \omega_{i}^{3} x_{i j \circ}^{3}+\sum_{d=1}^{D} \pi_{d}^{3} z_{d j \circ}^{3} ; \sum_{i=1}^{m} \omega_{i}^{1^{\prime}} x_{i j \circ}^{1^{\prime}}+\sum_{d=1}^{D} \pi_{d}^{1^{\prime}} z_{d j \circ}^{1^{\prime}}, \sum_{i=1}^{m} \omega_{i}^{2} x_{i j \circ}^{2}\right.\right. \\
& \left.\left.+\sum_{d=1}^{D} \pi_{d}^{2} z_{d j \circ}^{2}, \sum_{i=1}^{m} \omega_{i}^{3^{\prime}} x_{i j \circ}^{3^{\prime}}+\sum_{d=1}^{D} \pi_{d}^{3^{\prime}} z_{d j \circ}^{3^{\prime}}\right)\right)=E V((1,1,1 ; 1,1,1)), \quad j=1,2, \ldots, n, \\
& \pi_{d}^{3^{\prime}} \geq \pi_{d}^{3} \geq \pi_{d}^{2} \geq \pi_{d}^{1} \geq \pi_{d}^{1^{\prime}} \geq \varepsilon>0, \quad d=1, \ldots, D, \\
& \mu_{r}^{3^{\prime}} \geq \mu_{r}^{3} \geq \mu_{r}^{2} \geq \mu_{r}^{1} \geq \mu_{r}^{1^{\prime}} \geq \varepsilon>0, \quad r=1, \ldots, s, \\
& \omega_{i}^{3^{\prime}} \geq \omega_{i}^{3} \geq \omega_{i}^{2} \geq \omega_{i}^{1} \geq \omega_{i}^{1^{\prime}} \geq \varepsilon>0, \quad i=1, \ldots, m, \\
& u^{1}, u^{2} \text { free in sign. }
\end{aligned}
$$


Now, according to equation (8) for the expected value of TIFNs and Remark 1, model (19) can be rewritten into a linear programming model as follows:

$$
\begin{aligned}
E_{\circ}^{I}= & \max \left(\sum_{r=1}^{s}\left(\mu_{r}^{1^{\prime}} y_{r j \circ}^{1^{\prime}}+\mu_{r}^{1} y_{r j \circ}^{1}+4 \mu_{r}^{2} y_{r j \circ}^{2}+\mu_{r}^{3} y_{r j \circ}^{3}+\mu_{r}^{3^{\prime}} y_{r j \circ}^{3^{\prime}}\right)\right. \\
& \left.+\sum_{d=1}^{D}\left(\pi_{d}^{1^{\prime}} z_{d j \circ}^{1^{\prime}}+\pi_{d}^{1} z_{d j \circ}^{1}+4 \pi_{d}^{2} z_{d j \circ}^{2}+\pi_{d}^{3} z_{d j \circ}^{3}+\pi_{d}^{3^{\prime}} z_{d j \circ}^{3^{\prime}}\right)\right)+8 u^{1}+8 u^{2} \\
\text { s.t. } \quad & \sum_{d=1}^{D}\left(\pi_{d}^{1^{\prime}} z_{d j}^{1^{\prime}}+\pi_{d}^{1} z_{d j}^{1}+4 \pi_{d}^{2} z_{d j}^{2}+\pi_{d}^{3} z_{d j}^{3}+\pi_{d}^{3^{\prime}} z_{d j}^{3^{\prime}}\right)-\sum_{i=1}^{m}\left(\omega_{i}^{1^{\prime}} x_{i j}^{1^{\prime}}+\omega_{i}^{1} x_{i j}^{1}+4 \omega_{i}^{2} x_{i j}^{2}+\omega_{i}^{3} x_{i j}^{3}+\omega_{i}^{3^{\prime}} x_{i j}^{3^{\prime}}\right) \leq-8 u^{1}, \quad, j=1,2, \ldots, n, \\
& \sum_{r=1}^{s}\left(\mu_{r}^{1^{\prime}} y_{r j}^{1^{\prime}}+\mu_{r}^{1} y_{r j}^{1}+4 \mu_{r}^{2} y_{r j}^{2}+\mu_{r}^{3} y_{r j}^{3}+\mu_{r}^{3^{\prime}} y_{r j}^{3^{\prime}}\right)-\sum_{d=1}^{D}\left(\pi_{d}^{1^{\prime}} z_{d j}^{1^{\prime}}+\pi_{d}^{1} z_{d j}^{1}+4 \pi_{d}^{2} z_{d j}^{2}+\pi_{d}^{3} z_{d j}^{3}+\pi_{d}^{3^{\prime}} z_{d j}^{3^{\prime}}\right) \leq 8 u^{2}, \quad j=1,2, \ldots, n, \\
& \sum_{i=1}^{m}\left(\omega_{i}^{1^{\prime}} x_{i j \circ}^{1^{\prime}}+\omega_{i}^{1} x_{i j \circ}^{1}+4 \omega_{i}^{2} x_{i j \circ}^{2}+\omega_{i}^{3} x_{i j \circ}^{3}+\omega_{i}^{3^{\prime}} x_{i j \circ}^{3^{\prime}}\right)+\sum_{d=1}^{D}\left(\pi_{d}^{1^{\prime}} z_{d j \circ}^{1^{\prime}}+\pi_{d}^{1} z_{d j \circ}^{1}+4 \pi_{d}^{2} z_{d j \circ}^{2}+\pi_{d}^{3} z_{d j \circ}^{3}+\pi_{d}^{3^{\prime}} z_{d j \circ}^{3^{\prime}}\right)=8, \quad j=1,2, \ldots, n, \\
& \pi_{d}^{3^{\prime}} \geq \pi_{d}^{3} \geq \pi_{d}^{2} \geq \pi_{d}^{1} \geq \pi_{d}^{1^{\prime}} \geq \varepsilon>0, \quad d=1, \ldots, D, \\
& \mu_{r}^{3^{\prime}} \geq \mu_{r}^{3} \geq \mu_{r}^{2} \geq \mu_{r}^{1} \geq \mu_{r}^{1^{\prime}} \geq \varepsilon>0, \quad r=1, \ldots, s, \\
& \omega_{i}^{3^{\prime}} \geq \omega_{i}^{3} \geq \omega_{i}^{2} \geq \omega_{i}^{1} \geq \omega_{i}^{1^{\prime}} \geq \varepsilon>0, \quad i=1, \ldots, m, \\
& u^{1}, u^{2} \text { free in sign. }
\end{aligned}
$$

So, solving model (20) gives the overall efficiency $E_{\circ}^{I}$ of each decision maker based on TIFNs.

In the following, the method of efficiency assessment of each stage of the two-stage structure based on TIFNs is explained.
The first case: if the efficiency of the first stage has a higher priority, according to model (15) of Chen et al. [35], the efficiency assessment model for the first stage will be as follows:

$$
\begin{aligned}
E_{\circ}^{1 *} & =\max \sum_{d=1}^{D} \pi_{d} z_{d j \circ}+u^{1}, \\
\text { s.t. } & \sum_{d=1}^{D} \pi_{d} z_{d j}+u^{1}-\sum_{i=1}^{m} \omega_{i} x_{i j} \leq 0, \quad j=1,2, \ldots, n, \\
& \sum_{r=1}^{s} \mu_{r} y_{r j}+u^{2}-\sum_{d=1}^{D} \pi_{d} z_{d j} \leq 0, \quad j=1,2, \ldots, n, \\
& \left(1-E_{\circ}\right) \sum_{d=1}^{D} \pi_{d} z_{d j \circ}+\sum_{r=1}^{s} \mu_{r} y_{r j o}+u^{1}+u^{2}=E_{\circ}, \quad j=1,2, \ldots, n, \\
& \sum_{i=1}^{m} \omega_{i} x_{i j o}=1, \quad j=1,2, \ldots, n, \\
& \pi_{d}, \mu_{r}, \omega_{i} \geq 0, \quad d=1, \ldots, D ; r=1, \ldots, s ; i=1, \ldots, m, \\
& u^{1}, u^{2} \text { free in sign, }
\end{aligned}
$$


where $E_{\circ}$ is the optimal value of model (15).
Using the same procedure followed for assessing overall efficiency (model (18)), the efficiency of the first stage based on TIFNs is obtained, in the following:

$$
\begin{aligned}
E_{\circ}^{1 *}= & \max \sum_{d=1}^{D}\left(\pi_{d}^{1^{\prime}} z_{d j \circ}^{1^{\prime}}+\pi_{d}^{1} z_{d j \circ}^{1}+4 \pi_{d}^{2} z_{d j \circ}^{2}+\pi_{d}^{3} z_{d j \circ}^{3}+\pi_{d}^{3^{\prime}} z_{d j \circ}^{3^{\prime}}\right)+8 u^{1}, \\
\text { s.t. } \quad & \sum_{d=1}^{D}\left(\pi_{d}^{1^{\prime}} z_{d j}^{1^{\prime}}+\pi_{d}^{1} z_{d j}^{1}+4 \pi_{d}^{2} z_{d j}^{2}+\pi_{d}^{3} z_{d j}^{3}+\pi_{d}^{3^{\prime}} z_{d j}^{3^{\prime}}\right)-\sum_{i=1}^{m}\left(\omega_{i}^{1^{\prime}} x_{i j}^{1^{\prime}}+\omega_{i}^{1} x_{i j}^{1}+4 \omega_{i}^{2} x_{i j}^{2}+\omega_{i}^{3} x_{i j}^{3}+\omega_{i}^{3^{\prime}} x_{i j}^{3^{\prime}}\right)+8 u^{1} \leq 0, \quad j=1,2, \ldots, n, \\
& \sum_{r=1}^{s}\left(\mu_{r}^{1^{\prime}} y_{r j}^{1^{\prime}}+\mu_{r}^{1} y_{r j}^{1}+4 \mu_{r}^{2} y_{r j}^{2}+\mu_{r}^{3} y_{r j}^{3}+\mu_{r}^{3^{\prime}} y_{r j}^{3^{\prime}}\right)-\sum_{d=1}^{D}\left(\pi_{d}^{1^{\prime}} z_{d j}^{1^{\prime}}+\pi_{d}^{1} z_{d j}^{1}+4 \pi_{d}^{2} z_{d j}^{2}+\pi_{d}^{3} z_{d j}^{3}+\pi_{d}^{3^{\prime}} z_{d j}^{3^{\prime}}\right)+8 u^{2} \leq 0, \quad j=1,2, \ldots, n, \\
& \left(1-E_{\circ}^{I}\right)\left(\sum_{d=1}^{D}\left(\pi_{d}^{1^{\prime}} z_{d j \circ}^{1^{\prime}}+\pi_{d}^{1} z_{d j \circ}^{1}+4 \pi_{d}^{2} z_{d j \circ}^{2}+\pi_{d}^{3} z_{d j \circ}^{3}+\pi_{d}^{3^{\prime}} z_{d j \circ}^{3^{\prime}}\right)\right)+\sum_{r=1}^{s}\left(\mu_{r}^{1^{\prime}} y_{r j \circ}^{1^{\prime}}+\mu_{r}^{1} y_{r j \circ}^{1}+4 \mu_{r}^{2} y_{r j \circ}^{2}+\mu_{r}^{3} y_{r j \circ}^{3}+\mu_{r}^{3^{\prime}} y_{r j \circ}^{3^{\prime}}\right) \\
& +8 u^{1}+8 u^{2}=8 E_{\circ}^{I}, \quad j=1,2, \ldots, n, \\
& \sum_{i=1}^{m}\left(\omega_{i}^{1^{\prime}} x_{i j \circ}^{1^{\prime}}+\omega_{i}^{1} x_{i j \circ}^{1}+4 \omega_{i}^{2} x_{i j \circ}^{2}+\omega_{i}^{3} x_{i j \circ}^{3}+\omega_{i}^{3^{\prime}} x_{i j \circ}^{3^{\prime}}\right)=8, \quad j=1,2, \ldots, n, \\
& \pi_{d}^{3^{\prime}} \geq \pi_{d}^{3} \geq \pi_{d}^{2} \geq \pi_{d}^{1} \geq \pi_{d}^{1^{\prime}} \geq \varepsilon>0, \quad d=1, \ldots, D, \\
& \mu_{r}^{3^{\prime}} \geq \mu_{r}^{3} \geq \mu_{r}^{2} \geq \mu_{r}^{1} \geq \mu_{r}^{1^{\prime}} \geq \varepsilon>0, \quad r=1, \ldots, s, \\
& \omega_{i}^{3^{\prime}} \geq \omega_{i}^{3} \geq \omega_{i}^{2} \geq \omega_{i}^{1} \geq \omega_{i}^{1^{\prime}} \geq \varepsilon>0, \quad i=1, \ldots, m, \\
& u^{1}, u^{2} \text { free in sign, }
\end{aligned}
$$

$$
\begin{aligned}
& w_{1}=\frac{\sum_{i=1}^{m} \omega_{i} x_{i j \mathrm{o}}}{\sum_{i=1}^{m} \omega_{i} x_{i j \mathrm{o}}+\sum_{d=1}^{D} \pi_{d} z_{d j \mathrm{o}}}, \\
& w_{2}=\frac{\sum_{d=1}^{D} \pi_{d} z_{d j \mathrm{o}}}{\sum_{i=1}^{m} \omega_{i} x_{i j \mathrm{o}}+\sum_{d=1}^{D} \pi_{d} z_{d j \mathrm{o}}} .
\end{aligned}
$$

To seek the appropriate weights of the first and second stages for the DMUs, which depend on the ratios of the inputs of each stage to all inputs of the structure, in the following, the parameters $w_{1}$ and $w_{2}$ are defined.

Note that $w_{1}$ and $w_{2}$ intended to represent the relative importance or contribution of the performances of the first and second stages, respectively, to the overall performance of the DMU. Suppose that $\sum_{i=1}^{m} \omega_{i} x_{i j_{\mathrm{o}}}+\sum_{d=1}^{D} \pi_{d} z_{d j \mathrm{o}}$ represent the total size of amount of resources consumed by the two-stage process, such that $\sum_{i=1}^{m} \omega_{i} x_{i j \mathrm{o}}$ and $\sum_{d=1}^{D} \pi_{d} z_{d j \mathrm{o}}$ are the sizes of the stages 1 and 2 , respectively. Then, the values of these parameters are given by relations (23), as follows:

Supposing that the optimum weights obtained from relations (23) are $w_{1}^{*}, w_{2}^{*}$, then the efficiency of the second stage is obtained by the following relation [35]:

$$
E_{\circ}^{2 *}=\frac{E_{\circ}^{*}-w_{1}^{*} \cdot E_{\circ}^{1^{*}}}{w_{2}^{*}} .
$$

The second case: if the efficiency of the second stage has a higher priority, then model (25) can be used to determine the efficiency of this stage: 


$$
\begin{array}{ll} 
& E_{\circ}^{2 *}=\max \sum_{r=1}^{s}\left(\mu_{r}^{1^{\prime}} y_{r j \circ}^{1^{\prime}}+\mu_{r}^{1} y_{r j \circ}^{1}+4 \mu_{r}^{2} y_{r j \circ}^{2}+\mu_{r}^{3} y_{r j \circ}^{3}+\mu_{r}^{3^{\prime}} y_{r j \circ}^{3^{\prime}}\right)+8 u^{2}, \\
\text { s.t. } \quad & \sum_{d=1}^{D}\left(\pi_{d}^{1^{\prime}} z_{d j}^{1^{\prime}}+\pi_{d}^{1} z_{d j}^{1}+4 \pi_{d}^{2} z_{d j}^{2}+\pi_{d}^{3} z_{d j}^{3}+\pi_{d}^{3^{\prime}} z_{d j}^{3^{\prime}}\right)-\sum_{i=1}^{m}\left(\omega_{i}^{1^{\prime}} x_{i j}^{1^{\prime}}+\omega_{i}^{1} x_{i j}^{1}+4 \omega_{i}^{2} x_{i j}^{2}+\omega_{i}^{3} x_{i j}^{3}+\omega_{i}^{3^{\prime}} x_{i j}^{3^{\prime}}\right)+8 u^{1} \leq 0, \quad j=1,2, \ldots, n, \\
& \sum_{r=1}^{s}\left(\mu_{r}^{1^{\prime}} y_{r j}^{1^{\prime}}+\mu_{r}^{1} y_{r j}^{1}+4 \mu_{r}^{2} y_{r j}^{2}+\mu_{r}^{3} y_{r j}^{3}+\mu_{r}^{3^{\prime}} y_{r j}^{3^{\prime}}\right)-\sum_{d=1}^{D}\left(\pi_{d}^{1^{\prime}} z_{d j}^{1^{\prime}}+\pi_{d}^{1} z_{d j}^{1}+4 \pi_{d}^{2} z_{d j}^{2}+\pi_{d}^{3} z_{d j}^{3}+\pi_{d}^{3^{\prime}} z_{d j}^{3^{\prime}}\right)+8 u^{2} \leq 0, \quad j=1,2, \ldots, n, \\
& \sum_{d=1}^{D}\left(\pi_{d}^{1^{\prime}} z_{d j \circ}^{1^{\prime}}+\pi_{d}^{1} z_{d j \circ}^{1}+4 \pi_{d}^{2} z_{d j \circ}^{2}+\pi_{d}^{3} z_{d j \circ}^{3}+\pi_{d}^{3^{\prime}} z_{d j \circ}^{3^{\prime}}\right)+\sum_{r=1}^{s}\left(\mu_{r}^{1^{\prime}} y_{r j \circ}^{1^{\prime}}+\mu_{r}^{1} y_{r j \circ}^{1}+4 \mu_{r}^{2} y_{r j \circ}^{2}+\mu_{r}^{3} y_{r j \circ}^{3}+\mu_{r}^{3^{\prime}} y_{r j \circ}^{3^{\prime}}\right) \\
& -E_{\circ}^{I}\left(\sum_{i=1}^{m}\left(\omega_{i}^{1^{\prime}} x_{i j \circ}^{1^{\prime}}+\omega_{i}^{1} x_{i j \circ}^{1}+4 \omega_{i}^{2} x_{i j \circ}^{2}+\omega_{i}^{3} x_{i j \circ}^{3}+\omega_{i}^{3^{\prime}} x_{i j \circ}^{3^{\prime}}\right)\right)+8 u^{1}+8 u^{2}=8 E_{\circ}^{I}, \quad j=1,2, \ldots, n, \\
& \sum_{d=1}^{D}\left(\pi_{d}^{1^{\prime}} z_{d j \circ}^{1^{\prime}}+\pi_{d}^{1} z_{d j \circ}^{1}+4 \pi_{d}^{2} z_{d j \circ}^{2}+\pi_{d}^{3} z_{d j \circ}^{3}+\pi_{d}^{3^{\prime}} z_{d j \circ}^{3^{\prime}}\right)=8, \quad j=1,2, \ldots, n, \\
& \pi_{d}^{3^{\prime}} \geq \pi_{d}^{3} \geq \pi_{d}^{2} \geq \pi_{d}^{1} \geq \pi_{d}^{1^{\prime}} \geq \varepsilon>0, \quad d=1, \ldots, D, \\
& \mu_{r}^{3^{\prime}} \geq \mu_{r}^{3} \geq \mu_{r}^{2} \geq \mu_{r}^{1} \geq \mu_{r}^{1^{\prime}} \geq \varepsilon>0, \quad r=1, \ldots, s, \\
& \omega_{i}^{3^{\prime}} \geq \omega_{i}^{3} \geq \omega_{i}^{2} \geq \omega_{i}^{1} \geq \omega_{i}^{1^{\prime}} \geq \varepsilon>0, \quad i=1, \ldots, m, \\
& u^{1}, u^{2} \text { free in sign, }
\end{array}
$$

where $E_{\circ}^{I}$ is the optimal value of model (20) and it is the overall efficiency too.

So, the same approach is taken to assess overall efficiency (model (20)), and the efficiency of the second stage based on TIFNs is obtained, as follows:

$$
E_{\circ}^{1 *}=\frac{E_{\circ}^{*}-w_{2}^{*} \cdot E_{\circ}^{2 *}}{w_{1}^{*}} .
$$

In the following, a numerical example is given to solve and evaluate the proposed method for an NDEA model with TIFN efficients.

\section{Numerical Example}

In this section, a numerical example is solved to illustrate how the proposed method and model determines efficiencies. In this example, there are 12 DMUs, such that each DMU is contained 3 inputs in the first stage, 2 intermediate products, and 3 outputs from the second stage. The schematic diagram of these DMUs is displayed in Figure 1.

The triangular intuitionistic fuzzy data considered for the numerical example are provided in Tables 1-3.

After solving models (20), (22), and (24) with the Lingo software, the overall efficiency, the efficiency of the first stage, and the efficiency of the second stage for 12 DMUs are obtained as shown in Table 4, as the efficiencies of a two-stage model of intuitionistic fuzzy data envelopment analysis (IFDEA).

The results of Table 4 show that the overall efficiency score of 6 DMUs is 1 . Also, for 7 DMUs of 12 DMUs, the efficiency score of the first stage is equal to 1 . However, for the second stage, 6 of the DMUs have an efficiency score of 1 . In fact, from 7 DMUs with an efficiency score of 1 in the first stage, only DMU9 has an efficiency score of less than 1 in the second stage. Among the evaluated units, DMU10 has the lowest overall efficiency score, which is 0.759 . This unit also has the lowest efficiency score in the first stage, which is 0.617 . In the second stage, the lowest efficiency score is 0.707 , which belongs to DMU9. Based on the obtained overall efficiency, first-stage efficiency, and second-stage efficiency scores, the compared DMUs can be classified into three groups listed in Table 5, where numbers 1 and 0 in this table are efficient and inefficient, respectively.

According to the classification in Table 5, five of the DMUs are completely inefficient, one of them is only efficient in the first stage, and six DMUs are efficient in both stages.

4.1. Comparison of the Efficiencies between a Two-Stage IFDEA Model and a Two-Stage DEA Model. To compare the results of the proposed model, the intuitionistic fuzzy data considered in the numerical example of this research are transformed to crisp numbers by relation (14).

Then, using models (15) and (21) and relation (24), Chen et al. [35], the overall efficiency and the efficiency of the first stage and the second stage for every 12 DMUs are obtained as shown in Table 6.

If the obtained results by the proposed model in Table 4 are compared with the obtained results in Table 6, it is observed that in the crisp model, 7 units are efficient in both stages, while in the proposed model, only 6 units are efficient in both stages. In other words, the differentiation between the units in the proposed model has been better. While the 9th unit, which has been efficient in both stages in the crisp model, has now been efficient only in the second stage for the proposed model. Finally, the proximity of the mean of the overall efficiency in the two methods $\left(\mu_{\mathrm{DEA}}=0.956, \mu_{\mathrm{IFDEA}}=0.938\right)$, indicated that the results of the proposed research model are correct and accurate. 


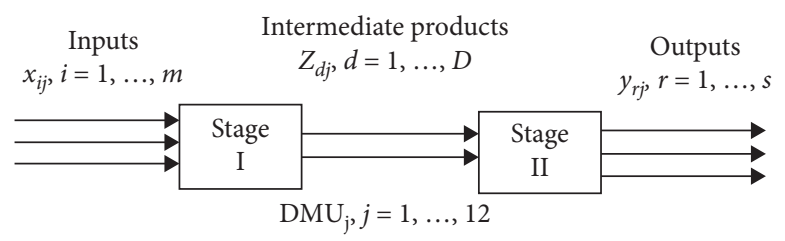

Figure 1: Schematic diagram of the two-stage DMUs considered in the example.

TABle 1: Triangular intuitionistic fuzzy inputs assumed for 12 DMUs.

\begin{tabular}{|c|c|c|c|}
\hline Input 3 & Input 2 & Input 1 & DMUs \\
\hline$(141,144,146 ; 138,144,147)$ & $(66,69,72 ; 64,69,74)$ & $(12,15,18 ; 10,15,20)$ & 1 \\
\hline$(125,127,130 ; 123,127,132)$ & $(28,32,35 ; 26,32,39)$ & $(14,18,21 ; 12,18,25)$ & 2 \\
\hline$(158,160,163 ; 154,160,165)$ & $(23,26,29 ; 21,26,31)$ & $(19,22,25 ; 17,22,27)$ & 3 \\
\hline$(160,163,165 ; 158,163,167)$ & $(33,37,39 ; 31,37,42)$ & $(20,24,26 ; 18,24,29)$ & 4 \\
\hline$(145,148,151 ; 143,148,153)$ & $(52,56,59 ; 50,56,61)$ & $(23,25,28 ; 21,25,32)$ & 5 \\
\hline$(232,235,238 ; 230,235,240)$ & $(67,71,74 ; 65,71,76)$ & $(42,46,49 ; 40,46,51)$ & 6 \\
\hline$(213,215,216 ; 210,215,218)$ & $(102,104,107 ; 100,104,109)$ & $(30,32,35 ; 28,32,38)$ & 7 \\
\hline$(202,206,208 ; 200,206,210)$ & $(131,133,135 ; 128,133,137)$ & $(29,31,34 ; 27,31,36)$ & 8 \\
\hline$(107,109,110 ; 105,109,112)$ & $(95,98,100 ; 92,98,102)$ & $(185,188,192 ; 181,188,194)$ & 9 \\
\hline$(261,267,270 ; 259,267,272)$ & $(165,168,172 ; 161,168,174)$ & $(46,49,53 ; 43,49,54)$ & 10 \\
\hline$(302,306,308 ; 300,306,309)$ & $(191,194,197 ; 189,194,199)$ & $(51,53,55 ; 48,53,57)$ & 11 \\
\hline$(279,282,284 ; 278,282,285)$ & $(205,208,211 ; 203,208,213)$ & $(32,36,38 ; 30,36,39)$ & 12 \\
\hline
\end{tabular}

Table 2: Triangular intuitionistic fuzzy intermediate products assumed for 12 DMUs.

\begin{tabular}{|c|c|c|c|}
\hline Output 3 & Output 2 & Output 1 & DMUs \\
\hline$(90,93,96 ; 88,93,98)$ & $(83,87,92 ; 81,87,94)$ & $(97,100,103 ; 95,100,105)$ & 1 \\
\hline$(68,70,71 ; 66,70,73)$ & $(43,45,48 ; 40,45,49)$ & $(147,149,150 ; 145,149,152)$ & 2 \\
\hline$(98,100,101 ; 96,100,103)$ & $(53,55,57 ; 50,55,59)$ & $(157,160,163 ; 154,160,165)$ & 3 \\
\hline$(112,114,117 ; 110,114,119)$ & $(60,62,64 ; 57,62,65)$ & $(170,172,175 ; 168,172,177)$ & 4 \\
\hline$(135,137,138 ; 133,137,140)$ & $(50,54,56 ; 48,54,59)$ & $(92,95,98 ; 90,95,100)$ & 5 \\
\hline$(177,180,183 ; 174,180,185)$ & $(77,80,83 ; 75,80,85)$ & $(225,227,228 ; 223,227,229)$ & 6 \\
\hline$(107,110,113 ; 105,110,115)$ & $(84,89,93 ; 82,89,96)$ & $(216,219,222 ; 214,219,224)$ & 7 \\
\hline$(108,110,111 ; 107,110,115)$ & $(94,99,103 ; 92,99,106)$ & $(215,218,221 ; 213,218,223)$ & 8 \\
\hline$(107,109,110 ; 105,109,112)$ & $(95,98,100 ; 92,98,102)$ & $(185,188,192 ; 181,188,194)$ & 9 \\
\hline$(151,153,155 ; 149,153,156)$ & $(96,99,103 ; 94,99,104)$ & $(245,249,252 ; 243,249,254)$ & 10 \\
\hline$(201,204,207 ; 198,204,209)$ & $(142,147,149 ; 140,147,152)$ & $(258,260,261 ; 256,260,262)$ & 11 \\
\hline$(126,129,132 ; 124,129,134)$ & $(118,119,122 ; 116,119,124)$ & $(247,249,255 ; 242,249,257)$ & 12 \\
\hline
\end{tabular}

Table 3: Triangular intuitionistic fuzzy outputs assumed for 12 DMUs.

\begin{tabular}{llc}
\hline Intermediate 2 & Intermediate 1 & DMUs \\
\hline$(79,83,88 ; 77,83,90)$ & $(99,102,104 ; 97,102,105)$ & 1 \\
$(39,41,44 ; 36,41,46)$ & $(101,103,106 ; 99,103,108)$ & 2 \\
$(21,22,25 ; 18,22,27)$ & $(81,84,86 ; 80,84,87)$ & 3 \\
$(31,32,35 ; 29,32,37)$ & $(91,94,96 ; 90,94,97)$ & 4 \\
$(57,61,64 ; 55,61,66)$ & $(121,124,126 ; 120,124,127)$ & 5 \\
$(79,82,85 ; 77,82,87)$ & $(220,222,223 ; 218,222,224)$ & 6 \\
$(85,90,94 ; 83,90,97)$ & $(111,114,116 ; 110,114,117)$ & 7 \\
$(88,91,93 ; 86,91,94)$ & $(143,146,148 ; 142,146,149)$ & 9 \\
$(113,115,116 ; 111,115,117)$ & $(167,169,170 ; 165,169,171)$ & 10 \\
$(93,97,102 ; 91,97,105)$ & $(115,117,118 ; 113,117,119)$ & 11 \\
$(223,225,228 ; 221,225,227)$ & $(135,137,138 ; 133,137,139)$ & 12 \\
$(216,219,222 ; 214,219,224)$ & $(171,173,176 ; 169,173,178)$ & \\
\hline
\end{tabular}


TABLE 4: The overall efficiency, the efficiency of the first stage, and the efficiency of the second stage of a two-stage IFDEA model.

\begin{tabular}{lccccc}
\hline$w_{2}^{*}$ & $w_{1}^{*}$ & $E_{\circ}^{2 *}$ & $E_{\circ}^{1 *}$ & $\begin{array}{c}\text { Total } \\
\text { efficiency }\end{array}$ & DMUs \\
\hline 0.498 & 0.502 & 1 & 1 & 1 & 1 \\
0 & 1 & 1 & 1 & 1 & 2 \\
0 & 1 & 1 & 1 & 1 & 3 \\
0.945464 & 0.054536 & 0.91273 & 0.8442226 & 0.9089938 & 4 \\
0.433431 & 0.566569 & 0.989237 & 0.9920930 & 0.9908552 & 5 \\
0.542585 & 0.457415 & 1 & 1 & 1 & 6 \\
0.387486 & 0.612514 & 0.977777 & 0.7418682 & 0.8332796 & 7 \\
0.521266 & 0.478734 & 0.920294 & 0.8776182 & 0.8998635 & 8 \\
0.467056 & 0.532944 & 0.707324 & 1 & 0.8633039 & 9 \\
0.412908 & 0.587092 & 0.959542 & 0.6171162 & 0.7585067 & 10 \\
0.4 & 0.6 & 1 & 1 & 1 & 11 \\
0.4983 & 0.5017 & 1 & 1 & 1 & 12 \\
\hline
\end{tabular}

TABle 5: Classification of the compared DMUs based on the obtained overall efficiency, first-stage efficiency, and second-stage efficiency scores.

\begin{tabular}{lrrcc}
\hline Category & $E_{\circ}^{1 *}$ & $E_{\circ}^{2 *}$ & Total efficiency & DMUs \\
\hline 1 & 1 & 0 & 1 & $\ldots$ \\
2 & 0 & 1 & 1 & $\ldots$ \\
3 & 1 & 1 & 1 & $1-2-3-6-11-12$ \\
4 & 0 & 0 & 1 & $\ldots$ \\
5 & 0 & 0 & 0 & $\ldots-5-7-8-10$ \\
6 & 1 & 1 & 0 & $\ldots$ \\
7 & 0 & 1 & 0 & 9 \\
8 & 1 & 0 & 0 & \\
\hline
\end{tabular}

TABle 6: The overall efficiency, and the efficiency of the first and the second stages of a two-stage DEA model.

\begin{tabular}{lccc}
\hline DMUs & Total efficiency & $E_{\circ}^{1 *}$ & $E_{\circ}^{2 *}$ \\
\hline 1 & 1 & 1 & 1 \\
2 & 1 & 1 & 1 \\
3 & 1 & 1 & 1 \\
4 & 0.881494 & 0.8924 & 0.8706 \\
5 & 0.996749 & 0.9940 & 0.9995 \\
6 & 1 & 1 & 1 \\
7 & 0.834047 & 0.7063 & 0.9618 \\
8 & 0.904673 & 0.8326 & 0.9767 \\
9 & 1 & 1 & 1 \\
10 & 0.85986 & 0.7966 & 0.9231 \\
11 & 1 & 1 & 1 \\
12 & 1 & 1 & 1 \\
\hline
\end{tabular}

\section{Conclusion}

The classic DEA models were designed to work with deterministic data and cannot deal with uncertainties in their inputs. The techniques so far developed for the assessment of inexact (fuzzy) efficiency are also very limited and, in some cases, defective. Given that inputs and outputs of real-world problems are not always deterministic and precise and some data can only be expressed in vague verbal and subjective terms and thus have a fuzzy or intuitionistic fuzzy nature, the use of fuzzy sets in mathematical modeling is imperative for overcoming with the challenges of dealing with such data.

Fuzzy sets are vague sets with imprecise boundaries, which were first introduced by Zadeh [16], which aimed to create a simpler model for complex systems. Following the development of fuzzy logic, intuitionistic fuzzy logic and fuzzy sets were introduced by Atanassov in 1983, as an extension to fuzzy logic [28]. Apart from a degree of membership, IFSs also have a degree of nonmembership. This leads to a decision matrix with a more accurate and reliable assessment and subsequently a more efficient and effective decision-making capability. Theory of IFS does not rule out the theory of fuzzy set and does not diminish its capabilities. Instead, it provides a more effective and efficient tool for dealing with uncertainty by using the extended form of fuzzy sets.

By the development of the application of intuitionistic fuzzy logic, several studies have used this logic in DEA approach. For example, Daneshvar et al. [50] in their study used a combination of intuitionistic fuzzy TOPSIS (IFTOPSIS) and DEA technique to evaluate decision-making units in both qualitative and quantitative terms. Edalatpanah [51] used the CCR model with TIFNs to evaluate the efficiency of decision-making units. In this method, based on the ranking function, the intuitionistic fuzzy model became a crisp linear programming model. In another study, Arya and Yadav [30] developed the CCR model with intuitionistic fuzzy data based on the alpha and beta cut method and obtained their dual. Also, Arya and Yadav [34] used the SBM model for IFNs and used the alpha and beta cut method to solve it. Otay et al. [32], by using the CCR model in the input oriented of the dual case and the addition operator for IFNs, presented a new hybrid approach consisting of AHP and DEA. Puri and Yadav [31] used the CCR model based on TIFNs and then transformed the model into linear programming with expected value. Hajiagha et al. [33] used interval IFNs and the BCC model in the dual case and used the generalized weighted operator to solve the model. Most studies in the field of DEA in IF environment are about a single-stage models. Among studies in network models, we can mention the study of Shakouri et al. [52]. They used the NDEA model in series and parallel structures. Then, by introducing the accuracy function, they have transformed the model into a crisp linear programming and ranked and evaluated the decision-making units based on the definition of alpha cut and degree of hesitation. These researchers in series structure, by using the data from Puri and Yadav's study [31], evaluated 16 hospital units using triangular fuzzy data for two inputs and two outputs. In parallel structure, they used the data of Ameri et al.'s study [53], for evaluating 8 hospital units, including 3 inputs with crisp data and 4 outputs with fuzzy TIFNs.

In another study, Ameri et al. [53] presented a model of NDEA in parallel structure and in the constant returns to scale for TIFNs and using the expected value transformed the model into a crisp linear programming model. Puri and Yadav [31], in their study, developed models to measure optimistic and pessimistic efficiencies of each DMU in intuitionistic fuzzy environment. By using superefficiency 
technique, algorithms are generated to obtain the complete ranking of the DMUs when optimistic and pessimistic situations are considered separately. In this paper, a hybrid of the IFDEA performance decision model is proposed, to address the overall performance using optimistic and pessimistic situations together in IF environments.

In this paper, Chen et al. [35] by using the developed model of the Kao and Hwang [36] presented a new two-stage model in variable returns to scale case and then by using expected value, the two-stage models of all intuitionistic fuzzy data became the crisp linear programming problem and deal with the evaluation of the performance of the units and their internal structures. This paper is a special type of the two-stage DEA model in variable returns to scale case in which all variables are expressed by TIFNs. Comparing the proposed model of the present study with the proposed model of Shakouri et al. [52], it can be stated that the proposed model of Shakouri et al. has not been used for all intuitionistic fuzzy data. The proposed two-stage model compared to Shakouri et al. has more power and application in the real world due to all intuitionistic fuzzy data and due to the intuitionistic fuzzy environment and two stages of the model, and the overall structure and each of the stages are separable and rankable which has made the model a unique advantage over other models. Also, in the proposed Puri and Yadav model [31], optimistic and pessimistic efficiency under the assumption of constant returns to scale as well as superefficiency and ranking of decision-making units for the initial models of DEA, i.e., the CCR model, in intuitionistic fuzzy environment are expressed. Although the proposed two-stage model in comparison with Puri and Yadav [31] is in the intuitionistic in fuzzy environment, it has paid attention to the structure of more than one stage, in which the source of inefficiency can be well identified and also in the studying of the structures of the two-stage models in intuitionistic fuzzy environment, the relationship between overall efficiency and the efficiency of the lower stages is less exposed to errors, and the optimal value of the intermediate variables is well determined.

Finally, in the proposed model of Ameri et al. [53], a selfassessment model of NDEA in intuitionistic fuzzy environment has been developed to measure the efficiency of the parallel system, at the time interval when some inputs and outputs are intuitionistic fuzzy in nature. While in the proposed model of the present study, due to all intuitionistic fuzzy data, the variables of the model including inputs and outputs and intermediates and weights can be evaluated in uncontrollable conditions.

In the end, the proposed model was used to solve a numerical example with 12 DMUs, containing 3 inputs in the first stage, 2 intermediate products, and 3 outputs in the second stage, using the Lingo software. Future studies are suggested to design multiplicative versions of two-stage DEA models with TIFNs.

\section{Data Availability}

The intuitionistic fuzzy number data used to support the findings of this study are based on the presentation of a new model, which have been included in the form of a numerical example in Tables 1-5 within the article.

\section{Conflicts of Interest}

The authors declare that there are no conflicts of interest regarding the publication of this paper.

\section{References}

[1] A. Charnes, W. W. Cooper, and E. Rhodes, "Measuring the efficiency of decision making units," European Journal of Operational Research, vol. 2, no. 6, pp. 429-444, 1978.

[2] A. I. Ali, C. S. Lerme, and L. M. Seiford, "Components of efficiency evaluation in data envelopment analysis," European Journal of Operational Research, vol. 80, no. 3, pp. 462-473, 1995.

[3] W. D. Cook and L. M. Seiford, "Data envelopment analysis (DEA)-thirty years on," European Journal of Operational Research, vol. 192, no. 1, pp. 1-17, 2009.

[4] J. Johnes, "Data envelopment analysis and its application to the measurement of efficiency in higher education," Economics of Education Review, vol. 25, no. 3, pp. 273-288, 2006.

[5] W. D. Cook, J. Zhu, G. Bi, and F. Yang, "Network DEA: additive efficiency decomposition," European Journal of Operational Research, vol. 207, no. 2, pp. 1122-1129, 2010.

[6] G. R. Jahanshahloo, A. Hadi Vencheh, A. A. Foroughi, and R. Kazemi Matin, "Inputs/outputs estimation in DEA when some factors are undesirable," Applied Mathematics and Computation, vol. 156, no. 1, pp. 19-32, 2004.

[7] M. Tavana and K. Khalili-Damghani, "A new two-stage Stackelberg fuzzy data envelopment analysis model," Measurement, vol. 53, pp. 277-296, 2014.

[8] R. Färe, S. Grosskopf, and G. Whittaker, Network DEA, Modeling Data Irregularities and Structural Complexities in Data Envelopment Analysis, Springer, Berlin, Germany, 2007.

[9] R. Färe, "Measuring Farrell efficiency for a firm with intermediate inputs," Academia Economic Papers, vol. 19, pp. 329-340, 1991.

[10] Y. Chen and J. Zhu, "Measuring information technology's indirect impact on firm performance," Information Technology and Management, vol. 5, no. 1-2, pp. 9-22, 2004.

[11] L.-F. Hsieh and L.-H. Lin, "A performance evaluation model for international tourist hotels in Taiwan-An application of the relational network DEA," International Journal of Hospitality Management, vol. 29, no. 1, pp. 14-24, 2010.

[12] Y. Chen, L. Liang, and J. Zhu, "Equivalence in two-stage DEA approaches," European Journal of Operational Research, vol. 193, no. 2, pp. 600-604, 2009.

[13] M. Maghbouli, A. Amirteimoori, and S. Kordrostami, "Twostage network structures with undesirable outputs: a DEA based approach," Measurement, vol. 48, pp. 109-118, 2014.

[14] T. R. Sexton and H. F. Lewis, "Two-stage DEA: an application to major league baseball," Journal of Productivity Analysis, vol. 19, no. 2-3, pp. 227-249, 2003.

[15] K. Tone, M. Tsutsui, and DEA "Network, "Network DEA: a slacks-based measure approach," European Journal of Operational Research, vol. 197, no. 1, pp. 243-252, 2009.

[16] L. A. Zadeh, "Fuzzy sets," Information and Control, vol. 8, no. 3, pp. 338-353, 1965.

[17] C. Kao and S. T. Liu, "Fuzzy efficiency measures in data envelopment analysis," Fuzzy Sets and Systems, vol. 113, pp. 427-437, 2000.

[18] S. Ramezanzadeh, A. Memariani, and S. Saati, "Data envelopment analysis with fuzzy random inputs and outputs: a chance-constrained programming approach," Iranian Journal of Fuzzy Systems, vol. 2, pp. 21-29, 2005. 
[19] S. M. Saati, A. Memariani, and G. R. Jahanshahloo, "Efficiency analysis and ranking of DMUs with fuzzy data," Fuzzy Optimization and Decision Making, vol. 1, pp. 255-267, 2002.

[20] S. Lertworasirikul, S. C. Fang, J. A. Joines, and H. L. Nuttle, "Fuzzy data envelopment analysis (DEA): a possibility approach," Fuzzy Sets and Systems, vol. 139, pp. 379-394, 2003.

[21] A. Emrouznejad and A. Mustafa, "Fuzzy data envelopment analysis: a discrete approach," Expert Systems with Applications, vol. 39, pp. 2263-2269, 2012.

[22] S. M. Mirhedayatian, S. E. Vahdat, M. J. Jelodar, and R. F. Saen, "Welding process selection for repairing nodular cast iron engine block by integrated fuzzy data envelopment analysis and TOPSIS approaches," Materials \& Design, vol. 43, pp. 272-282, 2013.

[23] P. Guo and H. Tanaka, "Fuzzy DEA: a perceptual evaluation method," Fuzzy Sets and Systems, vol. 119, no. 1, pp. 149-160, 2001.

[24] A. H. Ghapanchi, M. Tavana, M. H. Khakbaz, and G. Low, "A methodology for selecting portfolios of projects with interactions and under uncertainty," International Journal of Project Management, vol. 30, no. 7, pp. 791-803, 2012.

[25] S. A. H. Sadeghi, N. Ahmady, and E. Ahmady, "Technology selection in the presence of fuzzy data and dual-role factors," The International Journal of Advanced Manufacturing Technology, vol. 62, no. 5-8, pp. 801-811, 2012.

[26] M. Rostamy-Malkhalifeh and E. Mollaeian, "Evaluating performance supply chain by a new non-radial network DEA model with fuzzy data," Data Envelopment Analysis and Decision Science, vol. 2012, pp. 1-9, 2012.

[27] E. Houshyar, H. Azadi, M. Almassi, M. J. Sheikh Davoodi, and F. Witlox, "Sustainable and efficient energy consumption of corn production in Southwest Iran: combination of multifuzzy and DEA modeling," Energy, vol. 44, no. 1, pp. 672-681, 2012.

[28] K. T. Atanassov, "Intuitionistic fuzzy sets," Fuzzy Sets Ans Systems, vol. 20, no. 1, pp. 87-96, 1986.

[29] E. Szmidt and J. Kacprzyk, "Intuitionistic fuzzy sets in group decision making," Notes on IFS, vol. 2, no. 1, 1996.

[30] A. Arya and S. P. Yadav, “"Development of intuitionistic fuzzy data envelopment analysis models and intuitionistic fuzzy input-output targets," Soft Computing, pp. 1-19, 2018.

[31] J. Puri and S. P. Yadav, "Intuitionistic fuzzy data envelopment analysis: an application to the banking sector in India," Expert Systems with Applications, vol. 42, no. 11, pp. 4982-4998, 2015.

[32] İ. Otay, B. Oztaysi, S. Cevik Onar, and C. Kahraman, "Multiexpert performance evaluation of healthcare institutions using an integrated intuitionistic fuzzy AHP\&DEA methodology," Knowledge-Based Systems, vol. 133, pp. 90-106, 2017.

[33] S. H. R. Hajiagha, H. Akrami, E. Kazimieras Zavadskas, and S. S. Hashemi, "An intuitionistic fuzzy data envelopment analysis for efficiency evaluation under ucertainty: case of a finance and credit institution," Ekonomie a Management, vol. 16, no. 1, pp. 128-137, 2013.

[34] A. Arya and S. P. Yadav, "Development of intuitionistic fuzzy super-efficiency slack based measure with an application to health sector," Computers \& Industrial Engineering, vol. 115, pp. 368-380, 2018.

[35] Y. Chen, W. D. Cook, N. Li, and J. Zhu, "Additive efficiency decomposition in two-stage DEA," European Journal of Operational Research, vol. 196, no. 3, pp. 1170-1176, 2009.

[36] C. Kao and S.-N. Hwang, "Efficiency decomposition in twostage data envelopment analysis: an application to non-life insurance companies in Taiwan," European Journal of Operational Research, vol. 185, no. 1, pp. 418-429, 2008.

[37] K. Atanassov, "Intuitionistic fuzzy sets VII ITKR's Session," Sofia, June, vol. 1, p. 983, 1983.

[38] K. T. Atanassov, "More on intuitionistic fuzzy sets," Fuzzy Sets and Systems, vol. 33, no. 1, pp. 37-45, 1989.

[39] K. T. Atanassov, Intuitionistic Fuzzy Sets, Springer, Berlin, Germany, 1999.

[40] K. T. Atanassov, "Two theorems for intuitionistic fuzzy sets," Fuzzy Sets and Systems, vol. 110, no. 2, pp. 267-269, 2000.

[41] T. Buhaescu, "Some observations on intuitionistic fuzzy relations," in Itinerant Seminar of Functional Equations, Approximation and Convexity, pp. 111-118, 1989.

[42] A. I. Ban, "Nearest interval approximation of an intuitionistic fuzzy number," Computational Intelligence, Theory and Applications, pp. 229-240, Springer, Berlin, Germany, 2006.

[43] G. Deschrijver and E. Kerre, "On the relationship between intuitionistic fuzzy sets and some other extensions of fuzzy set theory," Journal of Fuzzy Mathematics, vol. 10, pp. 711-725, 2002.

[44] D. Stoyanova, "More on Cartesian product over intuitionistic fuzzy sets," BUSEFAL, vol. 54, pp. 9-13, 1993.

[45] G. Mahapatra and T. Roy, "Reliability evaluation using triangular intuitionistic fuzzy numbers arithmetic operations," World Academy of Science, Engineering and Technology, vol. 50, pp. 574-581, 2009.

[46] P. Grzegorzewski, "Distances and orderings in a family of intuitionistic fuzzy numbers," in Proceedings of the $3 \mathrm{rd}$ Conference of the European Society for Fuzzy Logic and Technology, pp. 223-227, Zittau, Germany, September 2003.

[47] S. K. Singh and S. P. Yadav, "Modeling and optimization of multi objective non-linear programming problem in intuitionistic fuzzy environment," Applied Mathematical Modelling, vol. 39, no. 16, pp. 4617-4629, 2015.

[48] R. Fare and S. Grosskopf, Intertemporal Production Frontiers, Dynamic DEA, Kluwer Academic, Boston, MA, USA, 1996.

[49] R. Färe and S. Grosskopf, "Network DEA," Socio-Economic Planning Sciences, vol. 34, no. 1, pp. 35-49, 2000.

[50] B. Daneshvar Rouyendegh, A. Yildizbasi, and I. Yilmaz, "Evaluation of retail industry performance ability through integrated intuitionistic fuzzy TOPSIS and data envelopment analysis approach," Soft Computing, pp. 1-12, 2020.

[51] S. A. Edalatpanah, "A data envelopment analysis model with triangular intuitionistic fuzzy numbers," International Journal of Data Envelopment Analysis, vol. 7, no. 4, pp. 47-58, 2019.

[52] B. Shakouri, R. Abbasi Shureshjani, B. Daneshian, and F. Hosseinzadeh Lotfi, "A parametric method for ranking intuitionistic fuzzy numbers and its application to solve intuitionistic fuzzy network data envelopment analysis models," Complexity, vol. 2020, Article ID 6408613, 25 pages, 2020.

[53] Z. Ameri, Sh. S. Sana, and R. Sheikh, "Self-assessment of parallel network systems with intuitionistic fuzzy data: a case study," Soft Computing, pp. 1-12, 2019. 